\title{
A STUDY OF BILINEAR MAPS IN WIRELESS SENSOR NETWORKS
}

\author{
Reza Alimoradi ${ }^{1}$, Seiied-Mohammad-Javad Razavian ${ }^{2}$ and Ali Ramzi ${ }^{3}$ \\ Department of Mathematics and Computer Science, University of Qom, Iran
}

\begin{abstract}
In the past, a large part of security requirements of wireless sensor networks (WSN) were fulfilled by symmetric cryptography systems. But, today by introduction of new needs in these networks and their security development, researchers of security and cryptography try to find new ways to increase efficiency and security of wireless sensor networks. Improving computational power of sensors used in wireless sensor networks made application of public key cryptography in WSN possible. Identity based cryptography is one important type of public key cryptography which using some bilinear functions called pairing functions was seriously applied. In this paper we will look at some researches done to find how to use pairings in wireless sensor networks.
\end{abstract}

\section{Keywords}

Wireless Sensor Network, Bilinear Function, Pairing, Identity Based Cryptography

\section{INTRODUCTION}

Wireless Sensor networks (WSN) are ad hoc networks which include a large number of small sensors and one or more base station(s). Due to their limited size and high cost, these sensors face limitations in energy consumption, memory and band width. WSN are used for gathering data and controlling the environment and are quite applicable in military or nonmilitary constructions such as battlefield monitoring, looking after the nature, traffic control and health care. They include a charged battery, a microprocessor and a radio transceiver. In recent years, in order to preserve security in WSN, symmetric cryptography systems like skipjack and RC5 were used for confidentially and identification. Symmetric cryptography systems are more appropriate for WSN compared to public key cryptography systems because they are more efficient in energy consumption and memory. However, key distribution and the number of saved keys are two main shortcomings of the symmetric cryptography systems. When unique keys are applied in WSN with $\mathrm{n}$ nodes, then each node must save ( $n-1)$ keys. Clearly, this is not efficient for large networks. Anyway, there will be no perfect forward security after disclosing a node's key. If a symmetric key is used, then the amount of memory needed will drastically decrease; but, in case of key exposure in one node, the whole network's security will be at risk. To remove this shortcoming, many possible key distribution schemes for the symmetric algorithm have been proposed. In general, all these schemes need pre-distributed keys which increase activities before development of the network. Therefore, asymmetric (public key) algorithms are quite valuable for key agreement and identification in WSN. Now, three types of key agreement schemes used generally in public networks will be introduced. One is a trusted server-based scheme which needs a trusted center for key agreement between the nodes. These schemes are not appropriate for WSN because of their lack of resources in energy and computations. Another scheme is based on pre-distribution of keys; it distributes key data before initiation and development of the network in all the nodes. Finally, a scheme based on public key in which cryptography is used. In the past, implementing public key cryptography on low-power systems, like sensor networks that use microprocessors and have many limitations, seemed very unlikely. Today, by making public key algorithms more efficient and also by increasing computational power of microprocessors, the farfetched dream of using public key cryptography has come true. Recently, many investigations have been done on WSN in order to make public key cryptography applicable [18]. For example, the results offered at $[8,17,18,23,30,32,33]$ show that elliptic curve cryptography (ECC) can be implemented on WSN. At present, elliptic curves are used in many portable systems like PDAs, smart cards, mobile phones and pagers. Sensors of TELOSB [5], MICAz, MICA2 [4] and Imote [3] families are appropriate for implementing public key cryptography. To apply ECC on WSN, attacks of a man in the middle must be prevented by public key identification. To achieve this, public key cryptography uses public key infrastructure (PKI). Clearly, implementing PKI needs a large amount of memory, computations and communications; that makes it inefficient for WSN. Using Identity based cryptography (IBC) can solve this problem. In this type of cryptography user's public identities like their email addresses or IPs can be used as their public key. Some papers in IBE have come in [9-13].Therefore, PKI will no longer be needed. After introduction of pairing based cryptography (PBC), this type of cryptography was applicably used. In fact, IBE seems to be the best solution to use public key cryptography on WSN. Examples of IBC offered for MANET are introduced in $[6,14,22,35,36]$. Instances of pairing based cryptography used in limited systems like WSN are also introduced in $[19,20,25,27,34]$.

In IBE systems, public identity of each WSN member node (sensor) i.e. each node's ID is considered that node's public key. Obviously, IBE systems need a trusted center to produce private key for the users and send it through a secure and private channel to them. In WSN, a base station (BS) can be responsible for key extract. Moreover, each node's private key can be uploaded inside each sensor before the network development. As IBE is more complicated than symmetric cryptography systems, thus, IBE is only used for production of a common key between two (or more) nodes. Public key based protocols for WSN are offered in [31]. These protocols include RSA-based identification and key agreement schemes which are named by Tiny PK. Tiny PK under $\mathrm{NesC}$ is implemented on MICAz 8-bit microprocessors. An exponentiation of RSA with 1024-bit key length can be done in 14.5 seconds; as a result, RSA- based structures are inappropriate for almost all applications such as WSN. Elliptic curve based cryptography (ECC) has a shorter key length compared to that of RSA (160 bits versus 1024 bits). Therefore, regarding the mentioned limitations for sensors based on 8-bit microprocessors, using ECC instead of RSA seems a lot more efficient [8]. The main action in ECC is scalar multiplication. In [17] a free software library named Tiny ECC is offered which is one of the fastest 
software libraries that apply ECC on WSN. It also supports all 128,160 and 192-bit standard curves of SECG [23]. Of course the software pack offered by SUN Microsystem Company which demands a high cost, is the fastest for this purpose.

Table.1. List of abbreviations

\begin{tabular}{|c|c|}
\hline Wireless Sensor Networks & WSN \\
\hline Elliptic Curve Cryptography & ECC \\
\hline Identity Based Cryptography & IBC \\
\hline Pairing Based Cryptography & PBC \\
\hline Public Key Infrastructure & PKI \\
\hline Base Station & BS \\
\hline Elliptic curve & $E$ \\
\hline Big prime numbers & $p, q$ \\
\hline Generator point $G_{1}$ of the order $q$ & $P$ \\
\hline Subgroups of the order $q$ & $G_{1}, G_{2}$ \\
\hline Distortion map & $\varnothing$ \\
\hline Multiplicative group of finite filed $F_{p^{2}}$ & $F_{p^{2}}$ \\
\hline Pairing function & $e, n_{T}$ \\
\hline
\end{tabular}

\section{MATHEMATICAL PRELIMINARIES}

Definition: Assuming $q$ is a prime number; if $G_{1}$ and $G_{2}$ are two cyclic groups of order $q$; it will be a pairing $e: G_{1} \times G_{2} \rightarrow G_{2}$ with these properties:

1. Bilinear: $e(a P, b Q)=e(P, Q)^{a b}$ for all $P, Q \in G_{1}, a, b \in Z_{q}^{*}$;

2. Non-Degenerate: There exists $P, Q \in G_{1}$, so that $e(P, Q) \neq$ 1 ;

3. Computable: There is an efficient algorithm to compute $e(P, Q)$ for all $P, Q \in G_{1}$.

Example: Assuming $q$ and $p$ are two prime numbers and $q \mid p^{2}-1$ and assuming $G_{1}$ and $G_{2}$ are two cyclic groups of the order $q$ when $G_{2}$ is a subgroup of $F_{p^{2}}^{*}$, the modified Weil pairing is this map $e$ : $G_{1} \times G_{2} \rightarrow G_{2}$ with above characteristics. If $\tilde{e}$ is a Weil pairing, then $e(P, P)=\tilde{e}(P, \varnothing(P))$, with $\varnothing$ as a distortion map.

Example: Assuming $E$ with the equation $y^{2}=x^{3}+1$ is defined over $F_{r}$ when $p=2(\bmod 3)$, therefore $E$ is super singular. w $\in F_{p^{2}}$ is considered as the third root of unity. Because $w$ 's order doesn't divide $F_{q}^{*}$ order; therefore, $w \notin F_{p}$. Now, we consider the isomorphism of $\begin{aligned} \varnothing: E\left(\bar{F}_{p}\right) & \rightarrow E\left(\bar{F}_{p}\right) \\ (x, y) & \rightarrow(w x, y)\end{aligned}$ and $\varnothing(\infty)=\infty$. Note that the order of the points $P$ and $\varnothing(P)$ is the same.

\section{ANALYZING EFFICIENCY OF THE SOFTWARE LIBRARY TINY PAIRING ON THE MICAZ SENSOR}

As mentioned above, the software pack called Tiny ECC is one of the fasted software libraries existed. So, many researchers compare results of their software implementations with this software as a touchstone. An instance is the software library called Tiny Pairing [34] which supports pairing functions and so is appropriate for pairing based cryptography (PBC). The Table.2 shows efficiency of the Tiny Pairing library [29] on the MICAz sensor. In the Table.2, 10 arbitrary inputs are selected for each action; then average time of the $n_{T}$ pairing is computed.

Table.2. Running time of some computations of PBC on MICAz by Tiny Pairing [27]

\begin{tabular}{|c|c|}
\hline & Time (sec) \\
\hline Hash-to-Point (16 bytes msg) & 0.89 \\
\hline Point compression & 0.38 \\
Point decompression & 0.38 \\
\hline Point Scalar multiplication & 7.75 \\
Point Scalar multiplication & 2.50 \\
Point Scalar multiplication & 2.45 \\
\hline$n_{T}$ pairing & 5.32 \\
\hline
\end{tabular}

Using these conclusions, there comes a comparison between implementation of some pairing based schemes by the Tiny Pairing with that of some elliptic curve based schemes by the Tiny ECC on the MICAz sensor.

Table.3. Implementation of some cryptography schemes on MICAz [34]

\begin{tabular}{|c|c|c|}
\hline Library & Tiny ECC & Tiny Pairing \\
\hline Scheme & ECIES & BF IBE \\
\hline Set up (sec) & - & 3.22 \\
\hline Key generation (sec) & - & 2.83 \\
\hline Encryption (sec) & 61.40 & 10.61 \\
\hline Decryption (sec) & 31.87 & 5.35 \\
\hline RAM (bytes) & 150 & 392 \\
\hline ROM (bytes) & 12,442 & 22,598 \\
\hline Size of public key/ID & $\begin{array}{c}160 \text { bit after } \\
\text { compressing }\end{array}$ & Arbitrary bit string \\
\hline
\end{tabular}

Table.4. Implementation of some signature schemes on MICAz [34]

\begin{tabular}{|c|c|c|c|}
\hline Library & Tiny ECC & \multicolumn{2}{|c|}{ Tiny Pairing } \\
\hline Scheme & ECDSA & BLS SS [1] & BBSS [2] \\
\hline Set up(sec) & 0 & - & - \\
\hline Key generation (sec) & - & 3.18 & 12.33 \\
\hline Signing (sec) & 30.72 & 4.08 & 3.0 \\
\hline Verification (sec) & 61.80 & 12.62 & 11.03 \\
\hline RAM (bytes) & 152 & 382 & 392 \\
\hline ROM (bytes) & 10,180 & 22,632 & 19,742 \\
\hline Size of signature (bit) & 320 & 160 & 312 \\
\hline
\end{tabular}




\section{COMPARING DIFFERENT TYPES OF SENSORS AND SOFTWARE PACKAGES}

The comparison is between different types of sensors like MICA2 which is a subset of MICAz and Imote2 and Sky Tmote. In this analysis two types of pairing functions $e(P, Q)$ and $n_{T}(P, Q)$ are implemented by software packs Nano ECC [26], Tiny Tate [19], Tiny PBC [20] and the one introduced in [27].

Table.5. implementation of pairings defined on $F_{p}, F_{2^{m}}$ for Imote 2 sensors [27]

\begin{tabular}{|c|c|c|c|c|}
\hline & \multicolumn{2}{|c|}{ Imote2(13MHz) } & \multicolumn{2}{c|}{ Imote2(104MHz) } \\
\hline Pairing & $n_{T}(P, Q)$ & $e(P, Q)$ & $n_{T}(P, Q)$ & $e(P, Q)$ \\
\hline Time & $0.46 \mathrm{~s}$ & $0.62 \mathrm{~s}$ & $0.06 \mathrm{~s}$ & $0.08 \mathrm{~s}$ \\
\hline ROM & $29.55 \mathrm{~KB}$ & $44.40 \mathrm{~KB}$ & $29.55 \mathrm{~KB}$ & $44.40 \mathrm{~B}$ \\
\hline Consumed energy & $12.12 \mathrm{~mJ}$ & $16.34 \mathrm{~mJ}$ & $3.76 \mathrm{~mJ}$ & $5.02 \mathrm{~mJ}$ \\
\hline
\end{tabular}

Table.6. Implementation of pairings defined on $F_{p}, F_{2^{m}}$ for Tmote Sky and MICAz sensors [27]

\begin{tabular}{|c|c|c|c|c|}
\hline & \multicolumn{2}{|c|}{ MICA2 } & \multicolumn{2}{c|}{ Tmote Sky } \\
\hline Parings & $n_{T}(P, Q)$ & $e(P, Q)$ & $n_{T}(P, Q)$ & $e(P, Q)$ \\
\hline Time & $2.66 \mathrm{~s}$ & $7.43 \mathrm{~s}$ & $1.71 \mathrm{~s}$ & $4.61 \mathrm{~s}$ \\
\hline ROM & $47.41 \mathrm{~KB}$ & $60.9 \mathrm{~KB}$ & $23.66 \mathrm{~KB}$ & $34.88 \mathrm{~B}$ \\
\hline Consumed energy & $62.73 \mathrm{~mJ}$ & $175.65 \mathrm{~mJ}$ & $17.70 \mathrm{~mJ}$ & $50.89 \mathrm{~mJ}$ \\
\hline
\end{tabular}

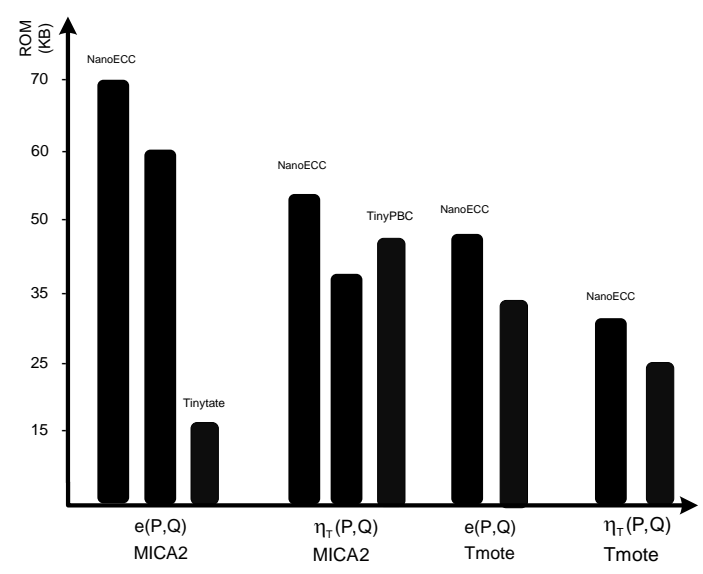

Fig.1. Comparing implementation of two pairings on some sensors regarding with ROM [27]

\section{ECDSA ALGORITHM OF THE SENSORS MICAZ, TELOSEB AND IMOTE}

Using selected curves secg defined on 128,160,192-bit prime fields, ECDSA algorithm is implemented. Sensors under experiment include MICAz, TeloseB and Imote2. The Table.7 Table.16 shows the results of implementing ECDSA on MICAz, TeloseB and Imote2.

Note that window length in scalar multiplication method is about $w=4$.

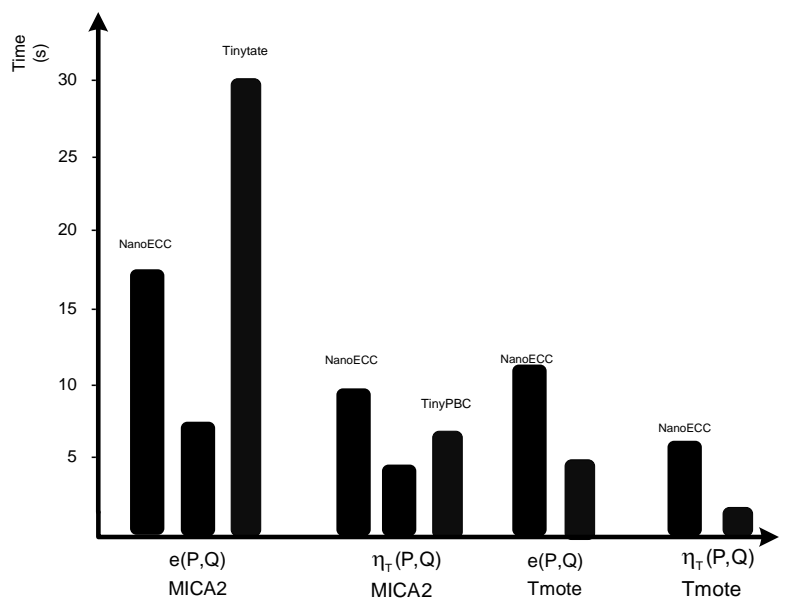

Fig.2. Comparing implementation of two pairings on some sensors regarding with time duration [27]

Table.7. Running time of ECDSA on MICAz for $w=4$ [15]

\begin{tabular}{|c|c|c|c|}
\hline Curve & Set up & Sign & Verification \\
\hline secp 128r1 & 2.522 & 1.923 & 2.418 \\
secp 128r2 & 2.518 & 2.069 & 2.674 \\
secp 160k1 & 3.553 & 2.059 & 2.441 \\
secp 160r 1 & 3.548 & 1.925 & 2.433 \\
secp 160r2 & 3.543 & 2.066 & 2.615 \\
secp 192k1 & 4.992 & 3.070 & 3.612 \\
secp 192r1 & 4.992 & 2.991 & 3.776 \\
\hline
\end{tabular}

Table.8. Running time of ECDSA on TeloseB for $w=4$ [15]

\begin{tabular}{|c|c|c|c|}
\hline Curve & Set up & Sign & Verification \\
\hline secp 128r1 & 3.861 & 4.059 & 5.056 \\
secp 128r2 & 3.847 & 4.325 & 5.618 \\
secp 160k1 & 5.208 & 4.433 & 5.209 \\
secp 160r1 & 5.225 & 4.361 & 5.448 \\
secp 160r2 & 5.197 & 4.457 & 5.609 \\
secp 192k1 & 7.190 & 6.695 & 7.840 \\
secp 192r1 & 7.204 & 6.651 & 8.331 \\
\hline
\end{tabular}

Table.9. Running time of ECDSA on Imote2 (104MHz) for $w=4$ [15]

\begin{tabular}{|c|c|c|c|}
\hline Curve & Set up & Sign & Verification \\
\hline secp 128r1 & 0.136 & 0.255 & 0.317 \\
secp 128r2 & 0.136 & 0.255 & 0.360 \\
secp 160k1 & 0.151 & 0.180 & 0.219 \\
secp 160r1 & 0.148 & 0.167 & 0.205 \\
secp 160r2 & 0.151 & 0.187 & 0.233 \\
secp 192k1 & 0.190 & 0.265 & 0.308 \\
secp 192r1 & 0.200 & 0.265 & 0.325 \\
\hline
\end{tabular}


Table.10. Running time of ECDSA on Imote2 (416 MHz) for $w=4[15]$

\begin{tabular}{|c|c|c|c|}
\hline Curve & Set up & Sign & Verification \\
\hline ecp 128r1 & 0.035 & 0.065 & 0.083 \\
secp 128r2 & 0.035 & 0.069 & 0.095 \\
secp 160k1 & 0.038 & 0.049 & 0.060 \\
secp 160r1 & 0.037 & 0.042 & 0.054 \\
secp 160r2 & 0.038 & 0.047 & 0.060 \\
secp 192k1 & 0.050 & 0.067 & 0.079 \\
secp 192r1 & 0.050 & 0.068 & 0.084 \\
\hline
\end{tabular}

The Table.10 shows energy required for computing ECDSA on these 3 sensors for a specific curve and various window lengths.

Table.11. Required energy to compute ECDSA for the curve secp160r1 [15]

\begin{tabular}{|c|c|c|c|c|}
\hline \multirow{2}{*}{$w$} & \multicolumn{2}{|c|}{ MICAz } & \multicolumn{2}{c|}{ TeloseB } \\
\cline { 2 - 5 } & Sign & Verification & Sign & Verification \\
\hline 2 & 52.9 & 58.4 & 27.5 & 29.4 \\
4 & 46.2 & 58.4 & 23.5 & 29.4 \\
8 & - & - & - & - \\
\hline
\end{tabular}

Table.12. Required energy to compute ECDSA for the curve secp160r1 [15]

\begin{tabular}{|c|c|c|c|c|c|c|}
\hline \multirow{4}{*}{$\boldsymbol{w}$} & \multicolumn{5}{|c|}{ Imote2 } \\
\cline { 2 - 7 } & \multicolumn{2}{|c|}{$13 \mathbf{M H z}$} & \multicolumn{2}{|c|}{ 104MHz } & \multicolumn{2}{|c|}{$416 \mathrm{MHz}$} \\
\cline { 2 - 7 } & Sign & Verification & Sign & Verification & Sign & Verification \\
\hline 2 & 2.56 & 2.72 & 0.32 & 0.34 & 0.08 & 0.10 \\
4 & 2.19 & 2.72 & 0.28 & 0.34 & 0.07 & 0.09 \\
8 & - & - & 0.24 & 0.34 & 0.06 & 0.09 \\
\hline
\end{tabular}

Energy needed for signature stage and verification of ECDSA algorithm for secp160r1 curves on different sensors has briefly come in these charts.

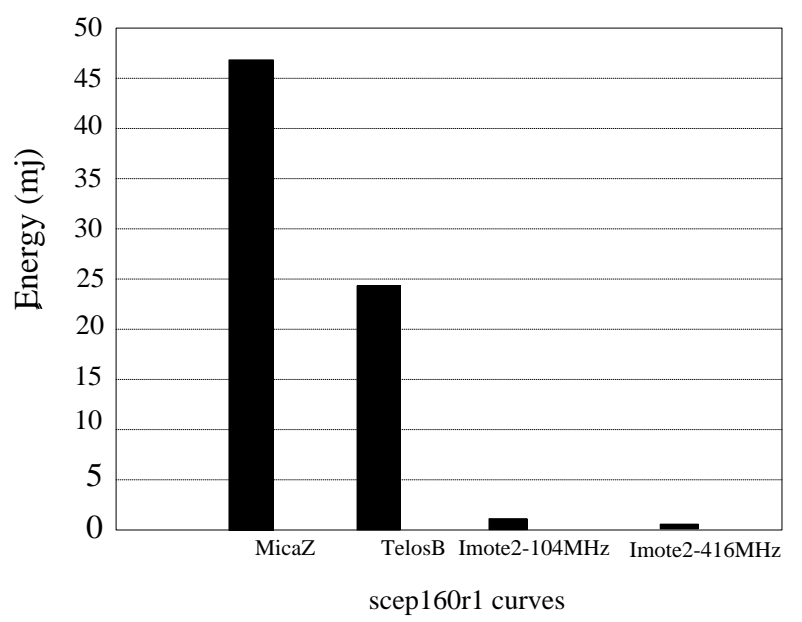

Fig.3. Required energy for the signature stage of ECDSA on some sensors [15]

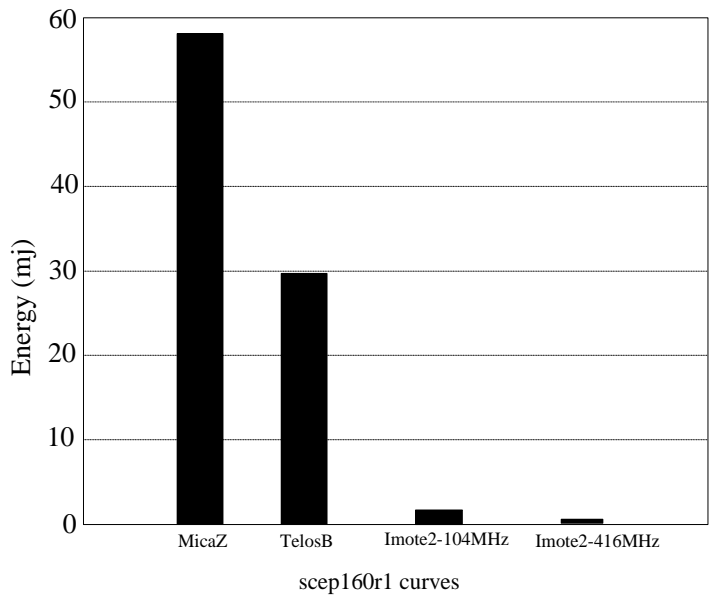

Fig.4. Required energy for the verification stage of ECDSA on some sensors [15]

Also, Time needed for the initiating stages and signing ECDSA algorithm for various curves on various sensors is explained here.

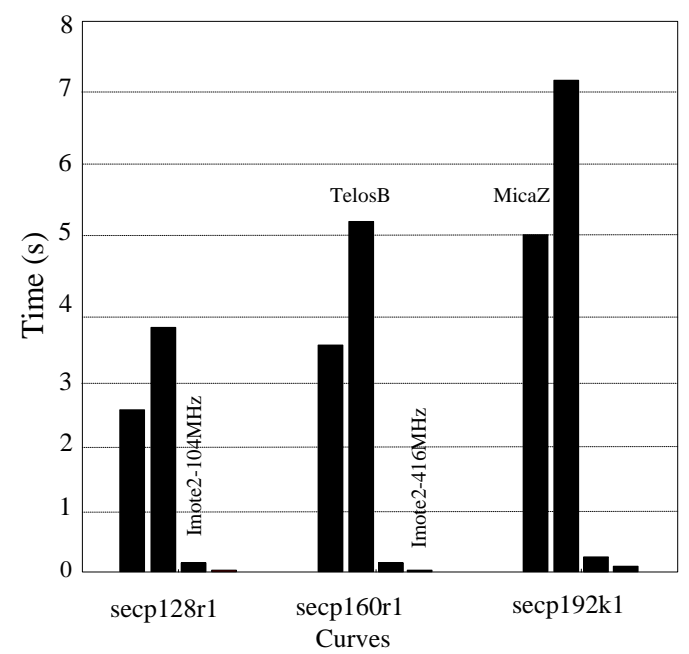

Fig.5. Running time of the initiative stage of ECDSA on some sensors [15]

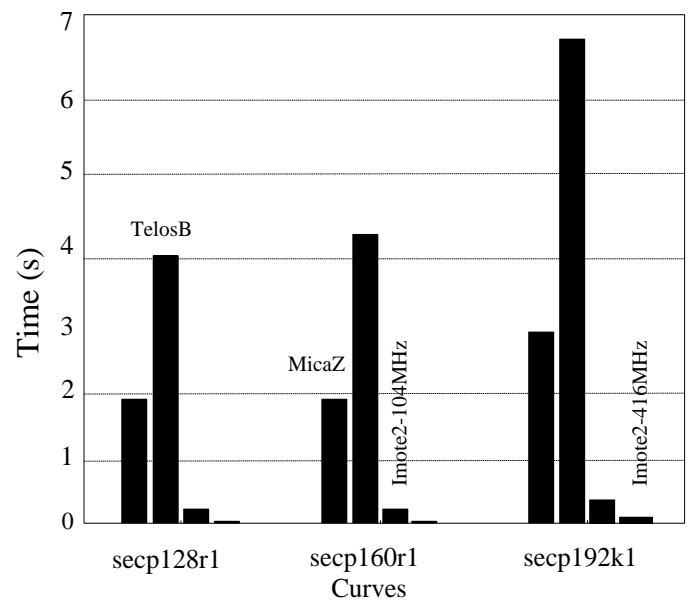

Fig.6. Running time of the signature stage of ECDSA on some sensors [15] 
These findings show the modern sensors of the type Imote2 are more efficient to implement elliptic curve based cryptography than MICAz and TeloseB sensors.

\section{IMPLEMENTING PAIRING FUNCTIONS ON MICAZ, TELOSEB AND IMOTE2 SENSORS}

As mentioned earlier, computing a pairing function like Tate function is expensive. Therefore Imote 2 is more efficient than MICAz and TeloseB. Here some results of the researches about implementing Tate function on Imote2 are explained. This analysis explicates size of the written program, time and energy required for implementing Tate function on Imote 2 for super singular curves defined on 192, 512-bit prime finite field. Size of the software program written on Imote2 for computing Tate function is explained in Table.12. As RAM memory of Imote2 is $32 \mathrm{MB}$, so, both curves for the size of the program are acceptable. This is also true about ROM memory.

Table.13. Size of program for computation of Tate pairing on Imote2 [15]

\begin{tabular}{|c|c|c|}
\hline Curve & ROM & RAM \\
\hline ss192k2 & 13,512 & 434 \\
ss512k2 & 13,844 & 1,034 \\
\hline
\end{tabular}

Table.14. Running time of Tate pairing on Imote2 (104MHz) [15]

\begin{tabular}{|c|c|c|c|}
\hline \multirow{2}{*}{ Curve } & \multicolumn{3}{|c|}{$104 M H z$} \\
\cline { 2 - 4 } & Miller & Final exponential & Sum \\
\hline ss192k2 & 0.459 & 0.032 & 0.491 \\
ss512k2 & 4.405 & 0.154 & 4.559 \\
\hline
\end{tabular}

Table.15. Running time of Tate pairing on Imote2 (416MHz) [15]

\begin{tabular}{|c|c|c|c|}
\hline \multirow{2}{*}{ Curve } & \multicolumn{3}{|c|}{$416 \mathrm{MHz}$} \\
\cline { 2 - 4 } & Miller & Final exponential & Sum \\
\hline ss192k2 & 0.115 & 0.008 & 0.123 \\
ss512k2 & 1.575 & 0.055 & 1.629 \\
\hline
\end{tabular}

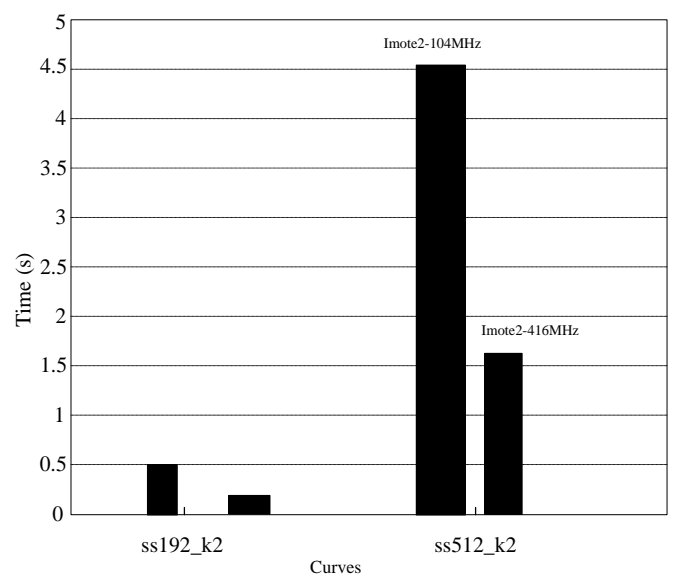

Fig.7. Running time of Tate pairing on Imote2 [15]
Computing Tate function has two main parts; one is miller algorithm and the other is exponentiation at the end of the Tate algorithm. Time required for running Tate algorithm on Imote 2 is shown in Table.12 and Table.13. With regard to time of running Tate algorithm for the 512- bit curve which contrary to the 192bit curve is more secure, this algorithm can be used in actual application of WSN. To prevent DoS attack which is a possible threat, projective coordinates can be used. This makes the computation time, 10 times faster. The coming table and chart show energy required for implementing Tate algorithm on Imote2.

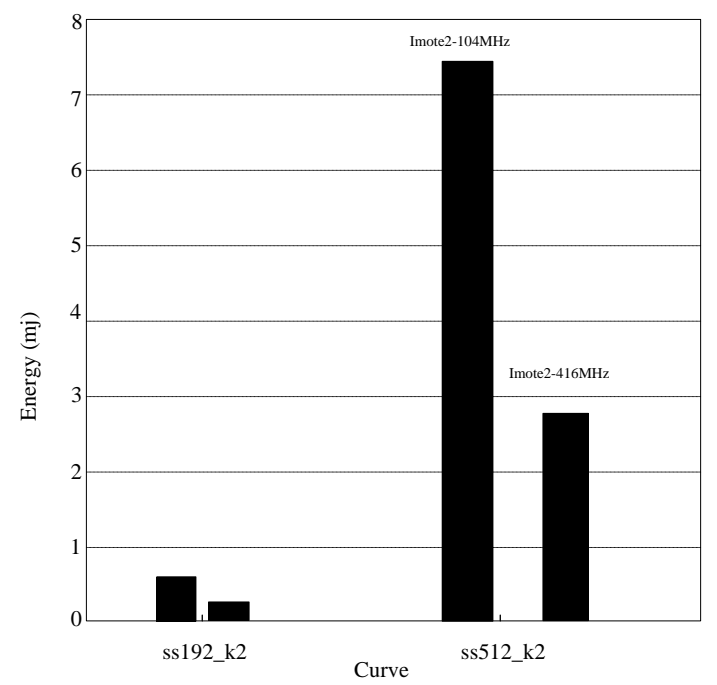

Fig.8. Energy consumption of Tate pairing on Imote2 [15]

Table.16. Energy consumption of Tate pairing on Imote2 [15]

\begin{tabular}{|c|c|c|}
\hline Curve & $\mathbf{1 0 4 M H z}$ & $\mathbf{4 1 6} \mathbf{M H z}$ \\
\hline ss192k2 & 0.80 & 0.20 \\
ss512k2 & 7.47 & 2.67 \\
\hline
\end{tabular}

Regarding the small amount of energy needed for computing Tate algorithm on Imote 2 compared to the energy needed for ECDSA algorithm verification on TeloseB and MICAz, thus, energy consumption of Tate algorithm on Imote 2 is acceptable.

\section{CONCLUSION}

Computing pairing functions is very expensive. Therefore, the powerful sensor Imote 2 is more efficient than MICAz and TloseB sensors. The pack introduced in [27] is functional than Nano ECC, Tiny Tate, Tiny PBC.

\section{REFERENCES}

[1] D. Boneh, H. Shacham and B. Lynn, "Short Signatures from the Weil Pairing", Proceedings of International Conference on Advances in Cryptology, pp. 514-532, 2001.

[2] D. Boneh and X. Boyen, "Short Signatures without Random Oracles and the SDH Assumption in Bilinear Groups", Journal of Cryptology, Vol. 21, No. 2, pp. 149-177, 2008.

[3] Crossbow Technology, "High-Performance Wireless Sensor Network Node", Available at: 
http://www.xbow.com/Products/Product_pdf_files/Wireles s_pdf/Imote2_Datasheet.pdf.

[4] Crossbow Technology, "Wireless Measurement System", Available

at: http://www.xbow.com/Products/Product_pdf_files/Wire less_pdf/MICAz_Datasheet.pdf.

[5] Crossbow Technology, "TELOSB Mote Platform", Available

at: http://www.xbow.com/Products/Product_pdf_files/Wire less_pdf/TelosB_Datasheet.pdf.

[6] H. Deng, A. Mukherjee and D. Agrawal, "Threshold and Identity-based Key Management and Authentication for Wireless Ad Hoc Networks", Proceedings of International Conference on Information Technology: Coding and Computing, 107-111, 2004.

[7] W. Diffiee and M. Hellman, "New Directions in Cryptography", IEEE Transactions on Information Theory, Vol. 22, No. 6, pp. 644-654, 1976.

[8] N. Gura, A. Patel, A. Wander, H. Eberle and S.C. Shantz, "Comparing Elliptic Curve Cryptography and RSA on 8-bit CPUs", Proceedings of $6^{\text {th }}$ International Workshop on Cryptographic Hardware and Embedded Systems, pp. 119-132, 2004.

[9] M.H. Dehkordi and R. Alimoradi, "Zero-Knowledge Identification Scheme Based on Weil Pairing", Lobachevskii Journal of Mathematics, Vol. 30, No. 3, pp. 203-207. 2009.

[10] M.H. Dehkordi and R. Alimoradi, "A New Batch Identification Scheme", Discrete Mathematics, Algorithms and Applications, Vol. 1, No. 3, pp. 369-376, 2009.

[11] M.H Dehkordi and R Alimoradi, "Authenticated Key Agreement Protocol", China Communications, Vol. 7, No. 5, pp. 1-8, 2010.

[12] M.H Dehkordi and R Alimoradi, "Identity-Based Multiple Key Agreement Scheme", KSII Transactions on Internet and Information Systems, Vol. 5, No. 12, pp. 2392-2402, 2011.

[13] M. H Dehkordi and R Alimoradi, "Certificateless Identification Protocols from Super Singular Elliptic Curve", Security and Communication Networks, Vol. 7, No. 6, pp. 979-986, 2014.

[14] K. Hoeper and G. Gong, "Identity-Based Key Exchange Protocols for Ad Hoc Networks", Proceedings of the Canadian Workshop on Information Theory, pp. 127-130, 2005.

[15] P. T. Kampanakis, "Identity-Based Cryptography: Feasibility and Applications in Next Generation Sensor Networks", Master of Science Thesis, North Carolina State University, 2007.

[16] A. Liu and P. Ning, "TinyECC: A Configurable Library for Elliptic Curve Cryptography in Wireless Sensor Networks", Proceedings of $7^{\text {th }}$ International Conference on Information Processing in Sensor Networks, pp. 245-256, 2008.

[17] J. Lopez, D. Aranha, D. Camara, R. Dahab, L. Oliveira and C. Lopes, "Fast Implementation of Elliptic Curve Cryptography and Pairing Computation for Sensor Networks", Proceedings of $13^{\text {th }}$ Workshop on Elliptic Curve Cryptography, pp. 117-121, 2009.

[18] D.J. Malan, M. Welsh and M.D. Smith, "Implementing Public-Key Infrastructure for Sensor Networks", ACM
Transactions on Sensor Networks, Vol. 4, No. 4, pp. 22-23, 2008.

[19] L.B. Oliveira, D.F. Aranha, E. Morais, F. Daguano, J. Lopez and R. Dahab, "TinyTate: Computing the Tate Pairing in Resource-Constrained Sensor Nodes", Proceedings of $6^{\text {th }}$ IEEE International Symposium on Network Computing and Applications, pp. 318-323, 2007.

[20] L.B. Oliveira, M. Scott, J. Lopez and R. Dahab, "TinyPBC: Pairings for Authenticated Identity-Based Non-Interactive Key Distribution in Sensor Networks", Proceedings of $5^{\text {th }}$ International Conference on Networked Sensing Systems, pp. 173-180, 2008.

[21] E. Ozturk, B. Sunar and E. Savascedil, "Low-Power Elliptic Curve Cryptography using Scaled Modular Arithmetic", Proceedings of International Workshop on Cryptographic Hardware and Embedded Systems, pp. 92-106, 2004.

[22] N. Saxena, G. Tsudik and J.H. Yi, "Identity-Based Access Control for Ad Hoc Groups", Proceedings of $7^{\text {th }}$ International Conference on Information Security and Cryptology, pp. 362-379, 2004.

[23] Standards for Efficient Cryptography Group, Available at: http://www.secg.org.

[24] S.C. Seo, D.G. Han and S. Song, "TinyECCK: Efficient Elliptic Curve Cryptography Implementation over GF $(2 \mathrm{~m})$ on 8-bit Micaz Mote", IEICE Transactions on Information and Systems, Vol. 91, No. 5, pp. 1338-1347, 2008.

[25] M. Shirase, Y. Miyazaki, T. Takagi, D.G. Han and D. Choi, "Efficient Implementation of Pairing Based Cryptography on a Sensor Node", IEICE Transactions on Information and Systems, Vol. 92, No. 5, pp. 909-917, 2009.

[26] P. Szczechowiak, L. Oliviera, M. Scott, M. Collier and R. Dahab, "NanoECC: Testing the limits of Elliptic Curve Cryptography in Sensor Networks", Proceedings of European Conference on Wireless Sensor Networks, Vol. 4913, pp. 305-320, 2008.

[27] P. Szczechowiak, A. Kargl, M. Scott and M. Collier, "On the Application of Pairing based Cryptography to Wireless Sensor Networks", Proceedings of $2^{\text {nd }}$ ACM Conference on Wireless Network Security, pp. 1-12, 2009.

[28] TinyOS, Available at: https://wiki2.org/en/TinyOS.

[29] TinyPairing library for wireless sensor networks, Available: http://www.cs.cityu.edu.hk/_ecc/TinyPairing.

[30] H. Wang and Q. Li, "Efficient Implementation of Public Key Cryptosystems on MICAz Motes", Proceedings of $8^{\text {th }}$ International Conference on Information and Communications Security, pp. 519-528, 2006.

[31] R. Watro, D. Kong, S.F. Cuti, C. Gardiner, C. Lynn and P. Kruus, "TinyPK: Securing Sensor Networks with Public Key Technology", Proceedings of $2^{\text {nd }}$ ACM Workshop on Security of Ad Hoc and Sensor Networks, pp. 59-64, 2004.

[32] T. Wollinger, J. Pelzl, V. Wittelsberger, C. Paar and G. Saldamli, "Elliptic and Hyper Elliptic Curves on Embedded Platform", ACM Transactions in Embedded Computing Systems, Vol. 3, No. 3, pp. 509-533, 2004.

[33] T. Wollinger, "Software and Hardware Implementation of Hyperelliptic Curve Cryptosystems", PhD Dissertation, Department of Electrical Engineering and Information Sciences, Ruhr University Bochum, 2004.

[34] X. Xiong, D.C. Wong, and X. Deng, "Tiny Pairing: Computing Tate Pairing on Sensor Nodes with Higher Speed 
and Less Memory", Proceedings of $8^{\text {th }}$ IEEE International Symposium on Network Computing and Applications, pp. 187-194, 2009.

[35] Y. Zhang, W. Liu, W. Lou and Y. Fang, "Securing Mobile Ad Hoc Networks with Certificateless Public Keys", IEEE
Transactions on Dependable and Secure Computing, Vol. 3, No. 4, pp. 386-399, 2006.

[36] Y. Zhang, W. Liu, W. Lou and Y. Fang, "Location-based Compromise-Tolerant Security Mechanisms for Wireless Sensor Networks", IEEE Journal on Selected Areas in Communications, Vol. 24, No. 2, pp. 247-260, 2006. 